\title{
Production of novel flower color mutants from the fragrant cyclamen (Cyclamen persicum $\times$ C. purpurascens) by ion-beam irradiation
}

\author{
Hiroshi Ishizaka*, Emiko Kondo, Naoko Kameari \\ Horticultural Laboratory, Saitama Prefecture Agriculture and Forestry Research Center, Kuki, Saitama 346-0037, Japan \\ *E-mail: ishizaka.hiroshi@pref.saitama.lg.jp Tel: +81-480-21-1113 Fax: +81-480-29-1021
}

Received December 26, 2011; accepted January 16, 2012 (Edited by Y. Ozeki)

\begin{abstract}
The fragrant cyclamens 'Uruwashi-no-kaori' (UR), 'Kaori-no-mai' (KM), 'Kokou-no-kaori' (KO) were bred from amphidiploids that were derived by chromosome doubling of the hybrid among Cyclamen persicum 'Strauss', 'Pure White' and fragrant wild species of C. purpurascens. Amphidiploid of GBCP was produced by chromosome doubling of the hybrid between C. persicum 'Golden Boy' and fragrant wild species of C. purpurascens. In order to create novel flower colors, mutation breeding with carbon-ion-beam irradiation was carried out using amphidiploids of UR, KM and KO as well as dihaploids of UR, KM and GBCP. Immature petals or etiolated petioles of the amphidiploids and dihaploids were irradiated, and plant tissue culture techniques were used to produce material for additional investigations. Furthermore, the stepwise improvement of flower color was achieved by irradiating 'ion3', which had been derived from dihaploid of UR by ion-beam irradiation. The mutants thus obtained were evaluated for breeding new cultivars of fragrant cyclamens. The tissues were then analyzed in terms of their flavonoid pigment content and their related genes.
\end{abstract}

Key words: Flower pigment, fragrant cyclamen, ion-beam, mutation, tissue culture.

The genus Cyclamen, family Myrsinaceae, includes 22 species. All species of Cyclamen form a tuber and consistently propagate by seed, but never propagate by natural splitting (Grey-Wilson 2002). Ornamental cyclamen cultivars, highly popular pot plants in several countries, have been developed through the crossing of selected natural mutants of wild C. persicum $(2 n=2 x=48)$. Flowers of $C$. persicum-derived cultivars may have a purple, pink, red, pale yellow or white 'slip', with an 'eye'; occasionally, the slip is pale yellow, pale pink or white and there is no 'eye'. 'Eye' refers to the bases of the petals colored deep purple and 'slip' refers to the other, non-eye parts of the petal, of various colors (Figure 1). Moreover, these flowers emit woody- or powdery-like scent, which has been expected to improve. In contrast, $C$. purpurascens $(2 n=2 x=34)$ has small flowers that consist of a purple slip and a deep purple eye, that emit a sweet fragrance, i.e. a rose-, hyacinth- or lily of the valley-like fragrance (Figure 1A) (Grey-Wilson 2002; Ishizaka et al. 2002). The ornamental value of the cultivars of $C$. persicum would be enhanced by the introduction of fragrance properties of $C$. purpurascens.
However, C. purpurascens has been neither improved as a major commercial cultivar nor used as breeding material.

In earlier work, an allodiploid $(2 n=2 x=41)$ was produced by the crossing of $C$. persicum cultivars $(2 n=2 x=48)$ and C. purpurascens $(2 n=2 x=34)$ through ovule culture techniques for rescuing abortive hybrid embryos, although all the allodiploids were sterile (Ishizaka and Uematsu 1995). In our subsequent study, however, fertile allotetraploids (amphidiploid, $2 n=4 x=82$ ) were successfully produced by inducing chromosome doubling with in vitro colchicine treatment of the hybrid ovules. Consequently, two types of fertile amphidiploid plant were produced by the following crosses: C. persicum 'Strauss' (Figure 1B) $\times$ C. purpurascens; C. persicum 'Pure White' (Figure 1C) $\times$ C. purpurascens. Three cyclamen cultivars emitting a fragrance similar to that of C. purpurascens, i.e. 'Uruwashi-no-kaori' (UR, Figure 1E), 'Kaori-no-mai' (KM, Figure 1F) and 'Kokou-no-kaori' (KO, Figure 1G), were subsequently bred by crossing these amphidiploids (Ishizaka 2008). Recently, fertile amphidiploid plant, named as GBCP (Figure $1 \mathrm{H}, 2 n=4 x=82$ ) was also

Abbreviations: Ch2G, chalcone 2-glucoside; CHI, chalcone isomerase; Cy3,5dG, cyanidin 3,5-diglucoside; Dp3,5dG, delphinidin 3,5-diglucoside; GBCP, amphidiploid between C. persicum 'Golden Boy' and C. purpurascens; KM, fragrant cyclamen cultivar-'Kaori-no-mai'; KO, fragrant cyclamen cultivar-'Kokou-no-kaori'; Mv3G, malvidin 3-glucoside; Mv3,5dG, malvidin 3,5-diglucoside; Pn3G, peonidin 3-glucoside; Pn3,5dG, peonidin 3,5-diglucoside; Pn3Nh, peonidin 3-neohesperidoside; UR, fragrant cyclamen cultivar-'Uruwashi-no-kaori'.

This article can be found at http://www.jspcmb.jp/

Published online June 20, 2012 

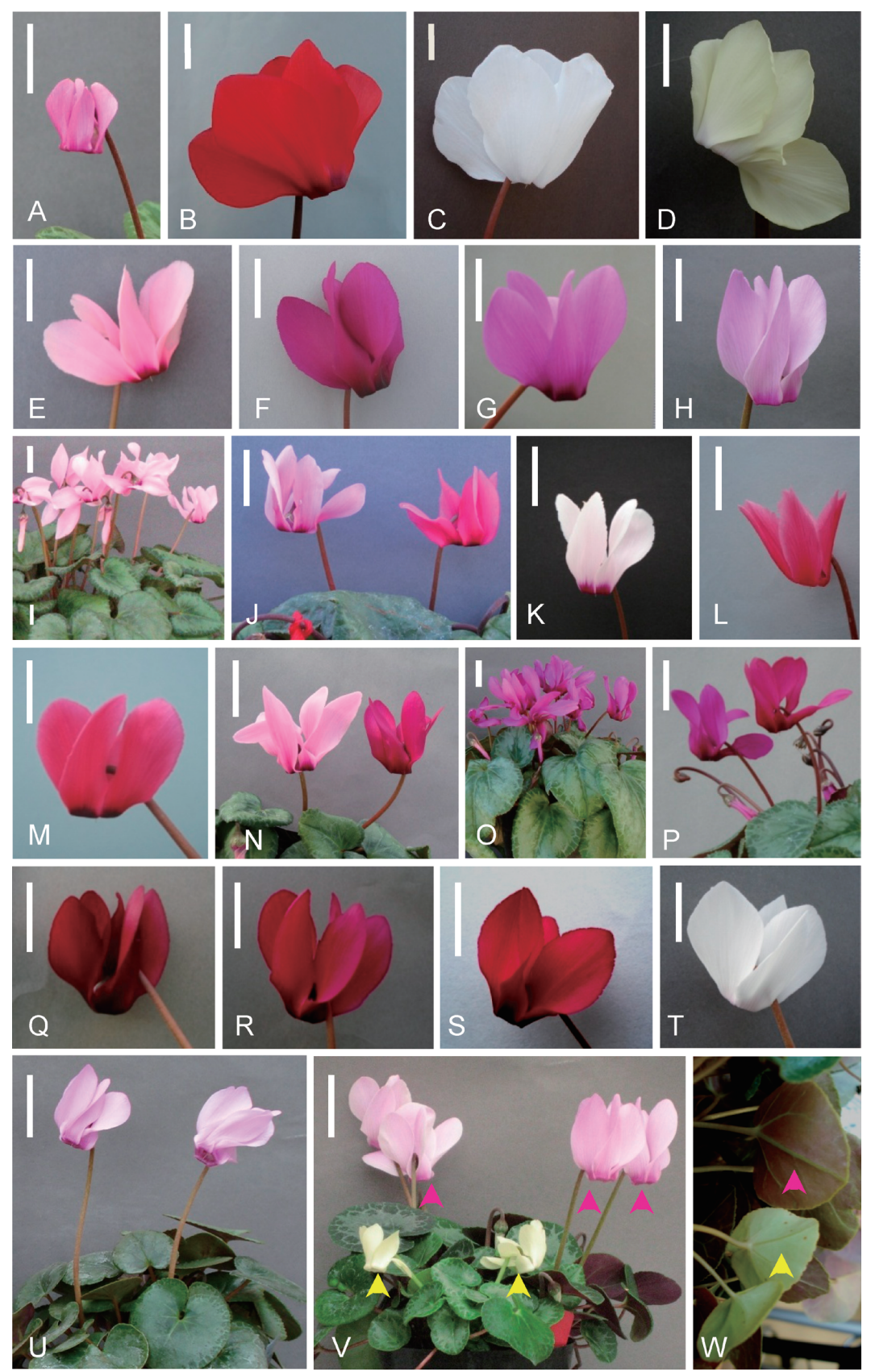

Figure 1. Flower coloration of the fragrant cyclamens, their parents and their ion beam-induced mutants. (A) Cyclamen purpurascens. (B) C. persicum 'Strauss'. (C) C. persicum 'Pure White'. (D) C. persicum 'Golden Boy'. (E) Amphidiploid of 'Uruwashi-no-kaori' (UR). (F) Amphidiploid of 'Kaori-no-mai' (KM). (G) Amphidiploid of 'Kokou-no-kaori' (KO). (H) Amphidiploid of C. persicum 'Golden Boy' $\times$ C. purpurascens (GBCP). (I) Dihaploid of 'Uruwashi-no-kaori' (UR). (J) Chimeric plant induced from the dihaploid of UR. Pink flower similar to that of the dihaploid of UR (left) and deeper-colored mutant (right, 'ion3'). (K) Lighter-colored mutant ('ion5') induced from the dihaploid of UR. (L) Mutant flower lacking the eye region ('ion255'), induced from 'ion3' re-irradiated with the ion beam. (M) Doubled-haploid mutant induced from 'ion3'. (N) Chimeric plant induced from the dihaploid of UR. Pink flower similar to that of the dihaploid of UR (left) and purple-flowered mutant (right). (O) Dihaploid of 'Kaori-nomai' (KM). (P) Chimeric plant induced from the dihaploid of KM. Purple flower similar to that of the dihaploid of KM (left) and red purple-colored mutant (right). (Q) Red purple-flowered mutant ('KMmv35') induced from the amphidiploid of KM. (R) Red purple-flowered mutant ('KMmv3') induced from the amphidiploid of KM. (S) Red purple-flowered mutant ('KMrp') induced from the amphidiploid of KM. (T) White flowered-mutant ('KOpw') induced from the amphidiploid of KO. (U) Dihaploid of GBCP. (V) Chimeric plant induced from the dihaploid of GBCP. Pale purple flowers similar to those of the dihaploid of GBCP (purple arrowheads) and yellow-colored mutant (yellow arrowheads). (W) Chimeric dihaploid plant of GBCP having leaves with original red-purple coloration (purple arrowhead), and mutated yellow-green coloration (yellow arrowhead) on the back, which are linked the original (V, purple arrowheads) and mutated (V, yellow arrowheads) flower colors, respectively. Scale bars are $20 \mathrm{~mm}$.

produced by inducing chromosome doubling of the hybrid, which had been obtained by crossing $C$. persicum 'Golden Boy' (Figure 1D, $2 n=2 x=48$ ) with C. purpurascens $(2 n=2 x=34)$ (Kameari et al. 2010a). 
Although C. persicum 'Strauss' (red flower), 'Pure White' (white flower) and 'Golden Boy' (pale yellow flower) were used as seed parents and C. purpurascens (purple flower) was used as the pollen parent in these interspecific hybridization, seed parent colors did not appear in the flowers of KO, UR, KM and GBCP.

Mutation breeding was chosen as the method to increase the variations in flower color of these fragrant cyclamens. For mutation breeding, gamma-rays or $\mathrm{X}$-rays have been often used to improve a target trait while retaining other traits (Yamaguchi 2001). As an alternative agent to gamma-rays or X-rays, ion beams have recently been used to induce novel mutants, a broad mutation spectrum and a high mutation rate because they can deposit a huge amount of energy in a limited area of the target genome (Tanaka et al. 2010). Accordingly, ion beams have been recently adopted for the mutation breeding of many commercial crops including cyclamens (Kondo et al. 2009c; Sugiyama et al. 2008; Tanaka et al. 2010). In this review, we discuss the induction of flower color mutants from the fragrant cyclamens by ion-beam irradiation in combination with plant tissue culture techniques. Flower pigments in the flavonoid biosynthetic pathway are the focus.

Ion-beam irradiation of dihaploids and amphidiploids of fragrant cyclamens in combination with tissue culture techniques

Dihaploid plants were produced from amphidiploids of UR, KM and GBCP by anther culture techniques described in previous reports (Kameari et al. 2010a; Kondo and Ishizaka 2003b), and they were held in the dark to induce etiolated petioles. The etiolated petioles reached about $50 \mathrm{~mm}$ in length were excised approximately $3 \mathrm{~mm}$ in length, and were irradiated with a $320 \mathrm{MeV}$ carbon-ion beam at doses ranging from 0 to $50 \mathrm{~Gy}$ under the culture condition, which were accelerated by a TIARA AVF cyclotron (JAEA, Takasaki, Japan). After the irradiation, plantlets were regenerated from the cultures through adventitious bud formation by the method described in previous reports (Kondo et al. 2003a; 2004; 2009a, b, c; 2010). $\mathrm{M}_{1}$ populations were established from regenerated plants of each irradiation, from which mutants were selected.

Flower color mutants were obtained from dihaploid of UR that had been irradiated at doses ranging from 0.1 to $10 \mathrm{~Gy}$. The $0.1 \mathrm{~Gy}$ dose gave rise to the mutant 'ion3', which was clonally propagated using tissue culture techniques. Then a stepwise improvement of flower color was performed again by irradiating 'ion3' with a $320 \mathrm{MeV}$ carbon-ion beam at doses ranging from 0 to $5 \mathrm{~Gy}$, and culturing selected mutants that arose (Kondo et al. 2009b).

Etiolated petioles of $\mathrm{KO}$ and $\mathrm{KM}$ were obtained by culturing their seeds aseptically in the dark. Immature petals before coloring were collected from buds of UR approximately $10 \mathrm{~mm}$ in length. The immature petals and etiolated petioles were irradiated with a $220 \mathrm{MeV}$ or $320 \mathrm{MeV}$ carbon-ion beam at doses ranging from 0 to 50 Gy under the culture condition. After the irradiation, plantlets were regenerated from the cultures through adventitious bud formation by using the previously described method (Kondo et al. 2003a; 2004; 2009a, b, c; 2010). Each irradiation dosage yielded an $M_{1}$ population of regenerated plants. $\mathrm{M}_{2}$ populations were established from individual, morphologically normal $\mathrm{M}_{1}$ plants, by self-pollination, and mutants were selected from the $\mathrm{M}_{2}$ populations.

Flower colors and pigments and flavonoid pathways of UR, KM, KO, GBCP and their parents

In cyanic cyclamens, 'Strauss' flowers have a red slip and a dark red eye, with the slip accumulating peonidin 3-glucoside (Pn3G) and peonidin 3-neohesperidoside (Pn3Nh) as its major anthocyanins, and the eye accumulating malvidin 3-glucoside (Mv3G). The slip and eye also accumulate quercetin and kaempferol as their major flavonols (Figure 1B) (Takamura et al. 2005; Webby and Boase 1999). Flowers of C. purpurascens with a purple slip and a deep purple eye accumulate malvidin 3,5-diglucoside $(\mathrm{Mv} 3,5 \mathrm{dG})$ along with quercetin and kaempferol (Figure 1A) (Takamura et al. 1997; 2005). In acyanic cyclamens, 'Pure White' accumulates quercetin and kaempferol into their white flowers as the major pigment (Figure 1C) (Miyajima et al. 1991; Takamura et al. 1995). Flowers of 'Golden Boy' accumulate chalcone 2-glucoside (Ch2G) into their pale yellow flowers as their major flower pigment, and also quercetin and kaempferol in trace amounts, suggesting that the biosynthetic pathway from chalcone to a flavonoid is inactivated (Figure 1D) (Miyajima et al. 1991; Sugimura et al. 1997). Furthermore, because 'Golden Boy' accumulates Ch2G in flowers as well as leaves, petioles and cotyledons, these organs are yellow green (Takamura et al. 1993; 1995).

Of the fragrant cyclamens, UR and its dihaploid have flowers with a pink slip accumulating cyanidin 3,5-diglucoside (Cy3,5dG), peonidin 3,5-diglucoside (Pn3,5dG) and Mv3,5dG and a dark red eye accumulating Mv3,5dG (Figure 1E, I). The slip and eye also accumulate quercetin and kaempferol (Kondo et al. 2009a). Flowers of KM and its dihaploid consist of a purple slip and a deep purple eye (Figure 1F, O), whereas those of KO have a light purple slip and a deep purple eye (Figure 1G). The slip and eye regions of $\mathrm{KM}$ and $\mathrm{KO}$ accumulate Mv3,5dG as their major anthocyanin along with quercetin and kaempferol as major flavonols (Kondo et al. 2009c; 2010). Flowers of GBCP and its dihaploid consist of a light purple slip and a purple eye that accumulate Mv3,5dG along with quercetin and kaempferol as major pigments (Figure 1H, 
The second branch

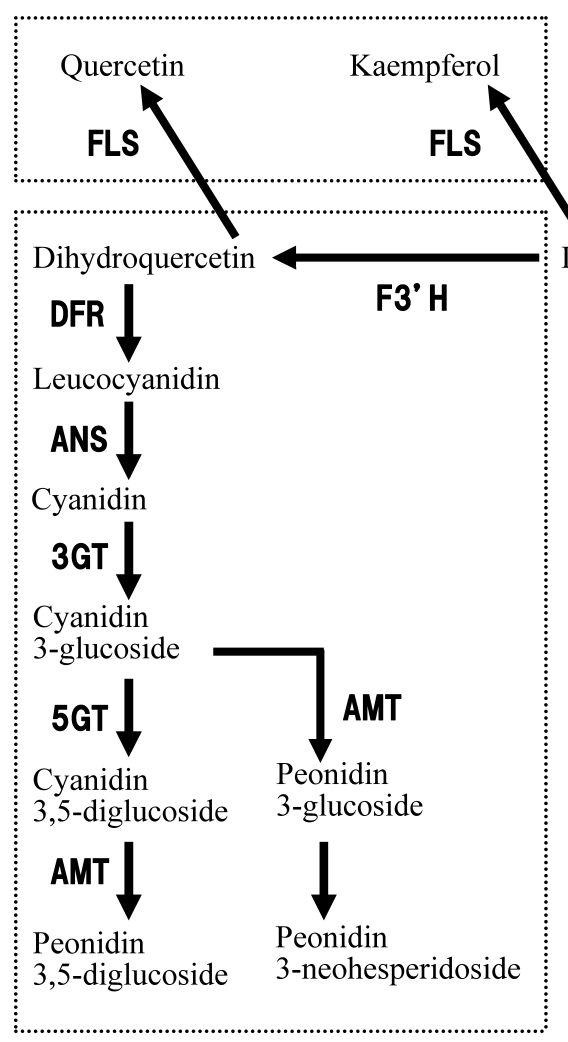

The third branch

4-Coumaroyl-CoA Malonyl-CoA

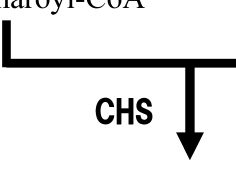

Chalcone

CHI

Naringenin

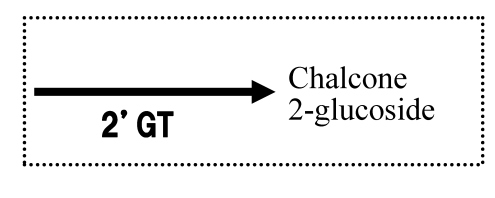

F3H

Dihydrokaempferol

DFR \}

Leucopelargonidin

ANS

Pelargonidin

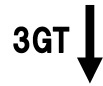

Pelargonidin 3-glucoside

5 GT

Pelargonidin

3,5-diglucoside

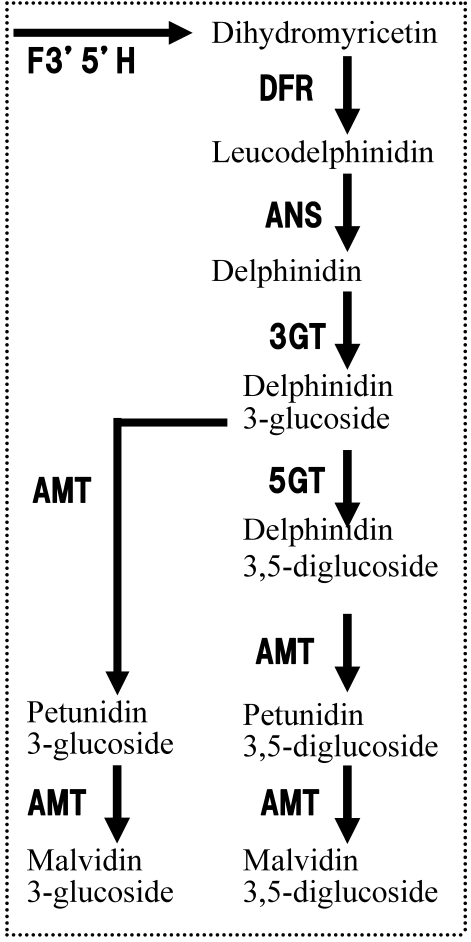

The first branch

Figure 2. Illustration of the flavonoid biosynthetic pathway in cyclamen plants. The pathway is divided broadly into two categories: the basic pathway and four branch pathways each enclosed in a dashed square. The branch pathway synthesizing pelargonidin 3,5-diglucoside is not enclosed in a dashed square, because cyclamen plants do not have the pathway. CHS, chalcone synthase; CHI, chalcone isomerase; $2^{\prime}$ GT, chalcone $2^{\prime}$-glucosyltransferase; F3H, flavone 3-hydroxylase; FLS, flavonol synthase; F3' H, flavonoid 3'-hydroxylase; F3' $5^{\prime} \mathrm{H}$, flavonoid 3' $5^{\prime}$-hydroxylase; DFR, dihydroflavonol 4-reductase; ANS, anthocyanidin synthase; 3GT, anthocyanidin 3-glucosyltransferase; 5GT, anthocyanin 5-glucosyltransferase; AMT, anthocyanin methyltransferase.

U), but they do not accumulate Ch2G (Kameari et al. 2010a; Takamura et al. 2005). Because of these patterns of anthocyanin accumulation in fragrant cyclamens and their parents, the accumulation of anthocyanin is thought to be regulated separately in the slip and eye regions.

The fragrant cyclamens are amphidiploids of the interspecific hybrids between $C$. persicum cvs. accumulating Pn3G, Pn3Nh or Mv3G as the major anthocyanin and C. purpurascens accumulating Mv3,5dG as the major anthocyanin. Therefore, it can be considered that anthocyanin 3,5-diglucoside accumulated in the fragrant cyclamens has been produced by glycosylation with 5-glucosyltransferase (5GT) originated from $C$. purpurascens, as reported by Takamura et al. (2005).
The flavonoid biosynthetic pathway consists of a basic pathway in which 4-coumaroyl-CoA is transformed to dihydrokaempferol and additional 'branch' pathways (Figure 2). The flavonoid biosynthetic pathway of carnations, petunias and cyclamens are described in Figure 2 of the Preface (Tanaka (2012) in this issue). In cyclamens, the first branch is where malvidinglucoside is synthesized from dihydromyricetin, a dihydrokaempferol derivative. In the second branch, quercetin- and kaempferol-glucosides are synthesized from dihydroquercetin and dihydrokaempferol, respectively and in the third branch, peonidinglucoside is synthesized from dihydroquercetin, a dihydrokaempferol derivative. In the fourth branch, chalcone-glucoside is synthesized through glycosylation. 
Almost all the wild species and cultivars have the first and/or second branches, while several cultivars have the second, third or fourth branches. By contrast, all the cyclamens including wild species and cultivars do not have a branch pathway to synthesize pelargonidinglucoside from dihydrokaempferol (Figure 2). Based on the biosynthesis pathways and the pigment distributions described above, the following events are presumed to have occurred in the parents of fragrant cyclamens. 'Strauss' has acquired the third branch in the flavonoid pathway by natural mutation of a wild species of $C$. persicum that has consistently retained the first and second branches. Genes involved in the second and third branches are expressed in the slip region, while those involved in the first and second branches are expressed in the eye. C. purpurascens has conserved genes involved in the first and second branches that are expressed in both the slip and eye. In 'Pure White', the genes involved in the second branch of the flavonoid pathway survived after the loss of genes involved in the first branch. 'Golden Boy' is a natural mutant of 'Pure White', which probably arose by a loss of the function of the chalcone isomerase (CHI) gene (Miyajima et al. 1991). Thus, 'Golden Boy' is thought to have acquired the fourth branch for disposing of surplus chalcone accumulated as the result of inactivation of $\mathrm{CHI}$ gene.

Based on the results given above, the following events are presumed to have occurred in the development of the fragrant cyclamens. UR has genes involved in the first, second and third branches of the flavonoid pathway. Genes involved in all three of those branches are expressed in the slip region and those involved in only the first and second branches are expressed in the eye region. $\mathrm{KM}$ and $\mathrm{KO}$ have genes involved in the first and second branches of the flavonoid pathway, which are expressed in both the slip and eye regions. GBCP has acquired the genes involved in the $\mathrm{CHI}$ and the first and second branches, probably originating from the $C$. purpurascens genome. These genes are expressed in both the slip and eye regions, resulting in synthesis of flavonols along with Mv3,5dG instead of Ch2G. Flower colors of UR, KM, KO and GBCP seem to be limited to pink and purple owing to Mv3,5dG, which is the end product of the first branch of $C$. purpurascens. Thus, it should be possible to increase the variations in flower color of fragrant cyclamens by regulating the genes involved in the first branch as well as the $C H I$ within the basic pathway.

\section{Mutation induction in dihaploid of UR}

Dihaploid of UR had flowers with a pink slip and a dark purple eye (Figure 1I), similar to the flowers of UR amphidiploid (Figure 1E). The dihaploid plants were irradiated with an ion beam at dosage raging from 0 to $50 \mathrm{~Gy}$. Two types of mutant were selected from the $\mathrm{M}_{1}$ population that had been irradiated with 0.1 and $2 \mathrm{~Gy}$ dosages: one was a chimeric plant that had both normal dihaploid flowers (Figure 1J, left) and mutant flowers, the latter having a salmon-colored slip and a dark purple eye (Figure 1J, right); the other had only mutant flowers with a pale pink slip and a purple eye (Figure 1K) (Kondo et al. 2008; 2009a). Dihaploid of UR and their mutants were sterile because of their haploidy. Accordingly, both mutants were cloned using tissue culture techniques and these clones were referred to as 'ion3' (Figure 1J, right) and 'ion5' (Figure 1K), respectively. The flower color of 'ion3' was deeper than that of the original dihaploid plants, whereas the flower color of 'ion5' was lighter than them. Both 'ion3' and 'ion5' accumulated Cy3,5dG, Pn3,5dG and Mv3,5dG in the slip region, and Mv3,5dG in the eye region, as their major anthocyanins. They also accumulated quercetin and kaempferol in both the slip and eye regions as their major flavonols. The constitution of these anthocyanins and flavonols in both the mutants was similar to that of UR dihaploid. The difference in coloration between 'ion3' and 'ion5' is related to anthocyanin and flavonol concentrations, which is discussed by Nakayama et al. (2012, in this issue).

To produce deepen flower color, 'ion3' was irradiated once again. As the result, two chimeric mutants with novel flower colors were selected from the $M_{1}$ population that had been irradiated with an ion-beam dosage of 0.5 and $1 \mathrm{~Gy}$. These chimeric mutants produced flowers with a salmon-red slip lacking a dark purple eye along with original flowers of 'ion3'. The mutant flowers were then cloned, and these clones were referred to as 'ion246' and 'ion255' (Figure 1L). Flowers of 'ion246' and 'ion255' accumulated Cy3,5dG, Pn3,5dG and Mv3,5dG as their major anthocyanins in the petals. The anthocyanin concentrations of these mutants were slightly higher than that of 'ion3' (Hase et al. (2012) in this issue). Because 'ion3', 'io246' and 'ion255' are dihaploids, they are completely sterile (Kondo et al. 2009b). We have started program for restoring the fertility of 'ion 246 ' and 'ion $255^{\prime}$ by inducing chromosome doubling to enable the development of new varieties.

Among the $M_{1}$ plants derived from 'ion3' after irradiating at dosages ranging from 0 to $10 \mathrm{~Gy}$, several plants with flowers larger than those of 'ion3' had restored fertility as a consequence of chromosome doubling (Figure 1M). This fact suggests that chromosome doubling occurred as a somaclonal variation at genome level, but influence of ion beam irradiation should also be considered as a causal factor (Kondo et al. 2011a). Fertile plants derived from plants receiving 0 Gy dose are probably candidates for new varieties if any variations caused by their mutation are not found in the following generation. Fertile plants derived from plants receiving 0.1 to $5 \mathrm{~Gy}$ dosages may yield some novel mutants by the effect of ion-beams in 
the following generation.

Two chimeric mutants with the same mutated flowers each other were also obtained from the $\mathrm{M}_{1}$ population of UR dihaploid irradiated at 0.5 and $10 \mathrm{~Gy}$ dosages. Their mutated flowers had a purple slip and dark purple eye region (Figure $2 \mathrm{~N}$, right) in comparison with the original flowers of the dihaploid (Figure 1I; 1N, left). The mutant flowers accumulated Mv3,5dG in the slip and eye as a major anthocyanin. Constitution of flower pigment in dihaploid of UR reveals that genes concerning the first branch are expressed in slip and eye regions, and those concerning the third branch are only expressed in slip region. The mutants with purple flower are thought to be induced by suppression of genes composing the third branch by ion-beam irradiation. Since flower color of the mutants closely resembled that of KM, we did not select this mutant for further investigation (Kondo unpublished data).

\section{Mutation induction in amphidiploid of UR}

A mutant with a salmon-colored slip and a dark red eye was obtained from the $\mathrm{M}_{2}$ population of UR amphidiploid irradiated with an ion-beam dosage of 2 Gy. The flowers accumulated Cy3,5dG, Pn3,5dG, Mv3,5dG, quercetin and kaempferol similar to those of 'ion3'. This mutant was selected for breeding a new fragrant cyclamen. Purple-flowered mutants, similar to those arising from UR dihaploid irradiated with an ion beam, were also obtained in the $\mathrm{M}_{2}$ population. They accumulated Mv3,5dG in the slip and eye as a major anthocyanin. Since flower color and morphological characteristics of these mutants closely resembled those of KM, we did not select these mutants as candidates for a new variety (Kondo unpublished data).

\section{Mutation induction in dihaploid of KM}

A chimeric mutant was obtained from the $\mathrm{M}_{1}$ population of KM dihaploid irradiated with an ion-beam dosage of $4 \mathrm{~Gy}$. Flowers of the KM dihaploid had a purple slip and a deep purple eye (Figure 1O) and accumulated Mv3,5dG, quercetin and kaempferol in the slip and eye. The chimeric mutant had both the original dihaploid flowers (Figure 1P, left) and mutant flowers having a red purple slip and a deep red purple eye (Figure 1P, right). The mutant flowers accumulated $\mathrm{Mv} 3 \mathrm{G}$ as a major anthocyanin as well as quercetin and kaempferol in the slip and eye, similar to those arising from KM amphidiploid irradiated with an ion-beam (Figure 1R) (Kameari et al. 2010b). Since flower color of the mutant closely resembled the flower color of the amphidiploidderived mutant (Figure 1Q, R, S), we did not clone the mutant for breeding new varieties.

\section{Mutation induction in amphidiploid of KM}

Amphidiploid of KM had flowers with a purple slip and a deep purple eye, and both regions accumulated Mv3,5dG as their major anthocyanin (Figure 1F). These were irradiated with ion-beam dosages ranging from 0 to $16 \mathrm{~Gy}$, and a mutant with flowers having a red-purple slip and a deep red-purple eye was selected from the $\mathrm{M}_{2}$ population that had been irradiated at 0.5 , 1 and $2 \mathrm{~Gy}$. The mutant induced by the $0.5 \mathrm{~Gy}$ dosage accumulated Mv3,5dG in the slip and eye as its major anthocyanin (KMmv35, Figure 1Q). Mutants from the 1 Gy dosage accumulated Mv3G in both the slip and eye (KMmv3, Figure 1R). These mutants are thought to result from inactivation of $5 \mathrm{GT}$, similar to the mutant from KM dihaploid. Mutants induced by $2 \mathrm{~Gy}$ of irradiation (KMrp, Figure 1S) accumulated delphinidin 3,5-diglucoside (Dp3,5dG) in both the slip and eye by inhibiting methylation of Dp3,5dG, and this clarified the causal gene of methylation (Akita et al. 2011; Kondo et al. 2009c; 2011b). Molecular aspects of methylation and glycosylation are described by Hase et al. (2012, in this issue). Since flowers of KMmv35, KMmv3 and KMrp are similarly colored, it is difficult to distinguish visually among them, although these flower colors obviously differ from those of KM. These findings suggest that coloration in these cyclamen mutants could be induced not only by a change in the chemical structure of anthocyanin pigments but also by the presence of flavonols as co-pigments. Expression of red-purple coloration in these mutants is discussed in relation to the flavonols as co-pigments by Nakayama et al. (2012, in this issue).

\section{Mutation induction in amphidiploid of $K O$}

Flowers of KO (Figure 1G) had a light purple slip and a deep purple eye, both of which accumulated Mv3,5dG, quercetin and kaempferol. Etiolated petioles of $\mathrm{KO}$ were irradiated with an ion beam at a dose of 0.1 and $0.2 \mathrm{~Gy}$, and white-flowered mutant, KOpw (Figure 1T), was selected from the $\mathrm{M}_{2}$ population that had been irradiated at a dose of $0.2 \mathrm{~Gy}$. KOpw accumulated quercetin and kaempferol as its major flavonols (Kondo et al. 2010), probably due to lack of Mv3,5dG, suggesting that genes involved in the first branch of the flavonoid biosynthetic pathway were affected by ion beams. In the genus Cyclamen, white-flowered variants exist in several wild species and in the C. persicum cultivar. The induction of white flowers in $\mathrm{KO}$ is discussed in relationship to the molecular analysis on white flowers of C. graecum album and C. persicum 'Pure White', in terms of a flower mutation map (Hase et al. (2012) in this issue).

\section{Mutation induction in dihaploid of GBCP}

Flowers of the dihaploid GBCP had a pale purple slip and a purple eye (Figure 1U), and accumulated Mv3,5dG along with quercetin and kaempferol. Irradiation with an ion-beam at dosages ranging from 0 to $5 \mathrm{~Gy}$ yielded an 
$\mathrm{M}_{1}$ population that involved chimeric plants with both mutant flowers with a pale yellow slip and eye (Figure $1 \mathrm{~V}$, yellow arrowheads) and flowers like those of the original dihaploid (Figure 1V, purple arrowheads). The pale yellow-flowered mutant accumulated Ch2G, while original pale purple flowers accumulated $\mathrm{Mv} 3,5 \mathrm{dG}$ (Nakayama, personal communication). Furthermore, in this pale yellow flower mutant, the leaves also expressed a yellow-green coloration on the back (Figure 1W, yellow arrowhead), whereas the leaves emerging from the region with pale purple original flowers expressed a red-purple coloration on the back (Figure 1W, purple arrowhead). Since the linkage between flower color and leaf color has been reported in cyanic cyclamen cultivars and 'Golden Boy' (Takamura et al.1993; 1995), change in leaf color would be a useful index for selecting mutants with yellow flowers in fragrant cyclamens. Molecular aspects of Ch2G and its related genes are described by Hase et al. (2012, in this issue). The etiolated petioles of mutant flowers were then cultured to regenerate plant, which was cloned and referred to as 'ion257'. Since 'ion257' is completely sterile because of its haploidy, we have started a program for restoring the fertility of 'ion 257 '.

\section{Conclusions}

Two types of regeneration pattern, adventitious bud formation and adventitious embryo formation are usually observed in in vitro culture of somatic tissues including cyclamen (Wicart et al. 1984); the former starts with multicellular tissue and the latter with a single cell (Carole et al.1993; Matsumoto et al. 1996). Accordingly, chimerism is expected to yield more frequently in the starting material consisting of more than one cell at the moment of mutagenic event. In our work, all regenerated plants after ion-beam irradiation were derived through the adventitious bud formation, and chimeric plants with the mutant flowers and flowers like those of the original plants were induced from the $M_{1}$ population of the dihaploids.

Monohaploid plant is useful for mutation breeding, compared with diploid plant, because genotypic change is directly expressed as phenotype due to the lacking one of the allele. In the dihaploids of UR, KM, GBCP and 'ion3', target genes should be present in chromosomes of both C. persicum and C. purpurascens, which have been expected to make the selection of mutants difficult. However, desirable mutants were successfully selected in $\mathrm{M}_{1}$ generation of these dihaploids. Therefore, interaction between genes of both species involved in phenotypic expression remains to be investigated. In amphidiploids of UR, KM and KO, the mutant flowers were not induced from the $M_{1}$ population, although they were induced from the $\mathrm{M}_{2}$ population. Thus, these mutant traits induced in $M_{1}$ and/or $M_{2}$ generation are probably recessive as described above in each section.
In the genus Cyclamen, all wild species, including $C$. persicum as the ancestral form of the current cultivars, consistently accumulate Mv3,5dG in their flowers through the function of the first branch in flavonoid biosynthetic pathway, except white-flowered variants (Figure 2). Since cultivation of the ancestral form of C. persicum has been initiated around 1700, two major breakthroughs for diversifying flower color and pigment have occurred through the discovery of natural mutants. Firstly, a red-flowered diploid mutant called 'Firefly' was discovered in 1886 (Grey-Wilson 2002), in which the mutation was possibly due to acquisition of the third branch in the flavonoid biosynthetic pathway. Secondly, a pale yellow-flowered diploid mutant 'Golden Boy' was discovered. This prototype of yellow flowered cyclamen arose through induction of the Ch2 $\mathrm{G}$ by a defect in the CHI gene (Miyajima et al. 1991). In our recent study, a third major breakthrough has occurred. Ion-beam irradiation of KM (amphidiploid of C. persicum $\times C$. purpurascens) containing $\mathrm{Mv} 3,5 \mathrm{dG}$ gave rise to an artificial mutant (KMrp) with a red-purple flower containing Dp3,5dG by a deletion of the AMT gene (Akita et al. 2011; Kondo et al. 2009c). Furthermore, ion-beam irradiation has yielded several candidates for new varieties: 'ion3', 'ion5', 'ion246', 'ion255', 'ion257', 'KMmv3', 'KMmv35' and 'KOpw'.

The fragrant cyclamens-UR, KM and $\mathrm{KO}$-released into the market around 2005 are a very small group, and differ genetically from commercial cultivars improved from C. persicum. In the future, the mutants produced from these fragrant cyclamens will be added to the fragrant cyclamen group as new varieties. We expect that the fragrant cyclamens will not only become a satisfactory group among the commercial cyclamens but also be used as parent materials for breeding of new varieties.

\section{Acknowledgments}

This research was supported by the "Research and development program for new bio-industry initiatives" from the Bio-oriented Technology Research Advancement Institution (BRAIN) of Japan and by the "Research project for utilizing advanced technologies" from the Ministry of Agriculture, Forestry and Fisheries (MAFF) of Japan.

\section{References}

Akita Y, Kitamura S, Hase Y, Narumi I, Ishizaka H, Kondo E, Kameari N, Nakayama M, Tanikawa N, Morita Y, Tanaka A (2011) Isolation and characterization of the fragrant cyclamen O-methyltransferase involved in flower coloration. Planta 234: $1127-1136$

Carole H, Saravitz, Frank A, Blazich, Amerson HV (1993) Histology of in vitro adventitious bud development on cotyledons and hypocotyls of fraser fir. J Am Soc Hortic Sci 118: $163-167$

Grey-Wilson C (2002) Cyclamen (A guide for gardeners, 
horticulturists and botanists). Timber Press, USA

Hase Y, Akita Y, Kitamura S, Narumi I, Tanaka A (2012) Development of an efficient mutagenesis technique using ion beams: Toward more controlled mutation breeding. Plant Biotechnol 29: 193-200

Ishizaka H (2008) Interspecific hybridization by embryo rescue in the genus Cyclamen. Plant Biotechnol 25: 511-519

Ishizaka H, Uematsu J (1995) Interspecific hybrids of Cyclamen persicum Mill. and C. purpurascens Mill. produced by ovule culture. Euphytica 82: 31-37

Ishizaka H, Yamada H, Sasaki K (2002) Volatile compounds in the flowers of Cyclamen persicum, C. purpurascens and their hybrids. Sci Hortic (Amsterdam) 94: 125-135

Kameari N, Kondo E, Nakayama M, Tanikawa N, Morita Y, Kurihara Y, Saotome T, Ishizaka H (2010a) Analysis of flower pigments and volatile compounds of hybrids between yellowflowered cultivar (Cyclamen persicum) and fragrant wild species (C. purpurascens). Breeding Res 12(suppl 1): 53, in Japanese

Kameari N, Nakayama M, Kondo E, Kurihara Y, Akita Y, Hase Y, Tanikawa N, Morita Y, Tanaka A, Ishizaka H (2010b) Analyses of flower pigments and volatile compounds of red-purple mutant generated by ion beam irradiation from haploid of fragrant purple cyclamen 'Kaori-no-mai' (Cyclamen persicum $\times C$. purpurascens). Hort Res (Japan) 9(suppl 2): 256, in Japanese

Kondo E, Ishizaka H (2003a) Adventitious bud formation from somatic tissues of F1 hybrid cyclamen (Cyclamen persicum $\times C$. purpurascens) and its amphidiploid. J Jpn Soc Hort Sci 72(suppl 2): 551, in Japanese

Kondo E, Ishizaka H (2003b) Plant regeneration from interspecific hybrid (Cyclamen persicum $\times$ C. purpurascens) and its amphidiploid by anther culture. J Jpn Soc Hort Sci 72(suppl 2): 550, in Japanese

Kondo E, Ishizaka H (2004) In vitro propagation of interspecific hybrid (Cyclamen persicum $\times$ C. purpurascens) and its amphidiploid by the use of adventitious embryos and etiolated petioles. J Jpn Soc Hort Sci 73(suppl 2): 582, in Japanese

Kondo E, Hase Y, Narumi I, Ishizaka H (2008) Mutation induction by ion-beam irradiation in fragrance cyclamen (Cyclamen persicum $\times$ C. purpurascens). JAEA-Review 055: 79, in Japanese with English abstract

Kondo E, Nakayama M, Takamura T, Kurihara Y, Saotome T, Hase Y, Tanaka A, Ishizaka H (2009a) Flower pigments and volatile compounds of mutant obtained from fragrance cyclamen (Cyclamen persicum $\times$ C. purpurascens) by ion beam irradiation. Hort Res (Japan) 8(suppl 2): 273, in Japanese

Kondo E, Kameari N, Nakayama M, Akita Y, Hase Y, Tanikawa N, Morita Y, Ishizaka H (2009b) Mutation induction in fragrant cyclamen (Cyclamen persicum $\times$ C. purpurascens) by ion beam reirradiation. Hort Res (Japan) 8(suppl 2): 274, in Japanese

Kondo E, Nakayama M, Kameari N, Tanikawa N, Morita Y, Akita Y, Hase Y, Tanaka A, Ishizaka H (2009c) Red-purple flower due to delphinidin 3,5-diglucoside, a novel pigment for Cyclamen spp., generated by ion-beam irradiation. Plant Biotechnol 26: 565-569

Kondo E, Nakayama M, Kameari N, Kurihara Y, Tanikawa N, Morita Y, Akita Y, Hase Y, Tanaka A, Ishizaka H (2010) Analyses of flower pigments and volatile compounds of white mutants generated by ion beam irradiation from fragrant purple cyclamen. 'Kokou-no-kaori' (Cyclamen persicum $\times C$. purpurascens). Hort Res (Japan) 9(suppl 2): 254, in Japanese
Kondo E, Kameari N, Kitamura S, Akita Y, Hase Y, Tanaka A, Nakayama M, Tanikawa N, Morita Y, Ishizaka H (2011a) Doubled haploid induction from haploid of fragrant cyclamen (Cyclamen persicum $\times$ C. purpurascens) by ion beam reirradiation. Hort Res (Japan) 10(suppl 2): 218, in Japanese

Kondo E, Nakayama M, Kameari N, Tanikawa N, Morita Y, Kitamura S, Akita Y, Hase Y, Tanaka A, Ishizaka H (2011b) Analysis of flower pigment of mutants generated from fragrant purple cyclamen 'Kaori-no-mai' (Cyclamen persicum $\times C$. purpurascens) by ion beam irradiation. Hort Res (Japan) 10(Suppl 2): 219, in Japanese

Matsumoto TK, Webb DT, Kuehnle AR (1996) Histology and origin of somatic embryos derived from Anthurium andraeanum Linden ex André Lamina. J Am Soc Hortic Sci 121: 404-407

Miyajima I, Maehara T, Kage T, Fujieda K (1991) Identification of the main agent causing yellow color of yellow-flowered cyclamen mutant. J Jpn Soc Hort Sci 60: 409-414

Nakayama M, Tanikawa N, Morita Y, Ban Y (2012) Comprehensive analyses of anthocyanin and related compounds to understand flower color change in ion-beam mutants of cyclamen (Cyclamen spp.) and carnation (Dianthus caryophyllus). Plant Biotechnol 29: 215-221

Sugimura T, Takamura T, Tanaka M (1997) Flower color and pigments in cyclamen cultivars. J Jpn Soc Hort Sci 66(Suppl 1): 410-411, in Japanese

Sugiyama M, Saito H, Ichida H, Hayashi Y, Ryuto H, Fukunishi N, Teraoka T (2008) Biological effects of heavy-ion beam irradiation on cyclamen. Plant Biotechnol 25: 101-104

Takamura T, Miyajima I, Maehara T (1993) Seedlings selection and micropropagation for the breeding of yellow-flowered cyclamen cultivars. J Fac Agr Kyushu Univ 37: 265-271

Takamura T, Tomihama T, Miyajima I (1995) Inheritance of yellowflowered characteristic and yellow pigments in diploid cyclamen (Cyclamen persicum Mill.) cultivars. Sci Hortic (Amsterdam) 64: 55-63

Takamura T, Omi S, Sugimura T, Tanaka M (1997) Flower color and anthocyanins in the petal of Cyclamen species. J Jpn Soc Hort Sci 66 (Suppl 2): 508-509, in Japanese

Takamura T, Nakayama M, Ishizaka H (2005) Inheritance of flower color pigment in crosses between cyclamen cultivars and Cyclamen purpurascens. Acta Hortic 673: 437-441

Tanaka A (2012) Targeted mutation breeding of flower color by taking advantage of ion-beam irradiation and genomic information. Plant Biotechnol 29: 191-192

Tanaka A, Shikazono N, Hase Y (2010) Studies on biological effects of ion beams on lethality, molecular nature of mutation, mutation rate, and spectrum of mutation phenotype for mutation breeding in higher plants. J Radiat Res (Tokyo) 51: 223-233

Webby RF, Boase MR (1999) Peonidin 3-O-neohesperidoside and other flavonoids from Cyclamen persicum petals. Phytochemistry 52: 939-941

Wicart G, Mouras A, Lutz A (1984) Histological study of organogenesis and embryogenesis in Cyclamen persicum Mill. tissue cultures: evidence for a single organogenetic pattern. Protoplasma 119: 159-167

Yamaguchi I (2001) Forty years of mutation breeding in Japan; research and fruits. Gamma Field Sym 40: 1-13 